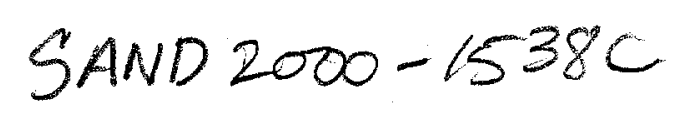

\title{
A Collective Collision Operator for DSMC
}

\author{
Michael A. Gallis and John R. Torczynski \\ Engineering Sciences Center. \\ Sandia National Laboratories" \\ Albuquerque New Mexico $87111-0826$ \\ USA
}

\begin{abstract}
A new scheme to simulate elastic collisions in particle simulation codes is presented. The new scheme aims at simulating the collisions in the highly collisional regime, in which particle simulation techniques typically become computationally expensive. The new scheme is based on the concept of a grid-based collision field. According to this scheme, the particles perform a single collision with the background grid during a time step. The properties of the background field are calculated from the moments of the distribution function accumulated on the grid. The collision operator is based on the Langevin equation. Based on comparisons with other methods, it is found that the Langevin method overestimates the collision frequency for dilute gases.
\end{abstract}

\section{INTRODUCTION}

Particle simulation methods and in particular the Direct Simulation Monte Carlo method (DSMC) have proved very successful in simulating complicated physical phenomena in a rarefied environment. However, many problems of practical interest involve dense flows that make DSMC simulation computationally very demanding, if not impossible.

Even in the most favorable problems, particle simulation methods have the disadvantage of being computationally expensive. The result is that the enormous potential of these methods in physical flow modeling has not been exploited by industry. Although massively parallel computers have contributed significantly in speeding up particle simulation codes, three-dimensional applications remain out of the scope of most modern computer platforms at present.

The most important limitation in modeling the high-density regime comes from the large number of collisions that particles undergo, which adds a very significant overhead to the calculation. In the case of non-reacting flows. these collisions keep reproducing the equilibrium distributions without adding any new information to the flow.

In a previous paper by Gallis and Torczynski (1), the BGK equation (2) and Cercignani's extension (3) were examined as possible collision operators. In this paper, a method based on the work of Langevin (4) to model the collisions in a collective manner is examined and compared with the previous methods.

\section{MODELS FOR THE COLLISION TERM OF THE BOLTZMANN EQUATION}

The complexity of the nonlinear structure of the collision integral of the Boltzmann equation has encouraged the development of simplified models that retain most of the properties of the Boltzmann equation but are not derived directly from it. The BGK method uses a simplified collision operator that takes the form:

$$
\left[\frac{\partial}{\partial t}(n f)\right]_{\text {collision }}=n v\left(f_{0}-f\right)
$$

\footnotetext{
"Sandia is a multiprogram laboratory operated by the Sandia Corporation, a Lockheed Martin Company, for the United States Department of Energy under Contract DE-AC04-94AL85000.
} 


\section{DISCLAIMER}

This report was prepared as an account of work sponsored by an agency of the United States Government. Neither the United States Government nor any agency thereof, nor any of their employees, make any warranty, express or implied, or assumes any legal liability or responsibility for the accuracy, completeness, or usefulness of any information, apparatus, product, or process disclosed, or represents that its use would not infringe privately owned rights. Reference herein to any specific commercial product, process, or service by trade name, trademark, manufacturer, or otherwise does not necessarily constitute or imply its endorsement, recommendation, or favoring by the United States Government or any agency thereof. The views and opinions of authors expressed herein do not necessarily state or reflect those of the United States Government or any agency thereof. 


\section{DISCLAIMER}

Portions of this document may be illegible in electronic image products. Images are produced from the best available original document. 
where $f_{o}$ is the Maxwellian distribution corresponding to the local temperature and average velocity, $f$ is the initial distribution of the particles, $n$ the number density, and $v$ is the collision frequency. Examination of the BGK model indicates that it reproduces the equilibrium solution $f=f_{0}$ at equilibrium. The BGK equation also reproduces the correct moments and satisfies the H-theorem. For the highly collisional regime, and when used with the ChapmanEnskog expansion, it leads to the Navier-Stokes equations. In this equation, the term $n v f_{o}$ represents the collisions replenishing the equilibrium distribution $f_{o}$, and the term $n v f$ represents the collisions depleting the distribution $f$.

The physical interpretation of the assumption that the replenishing number of collisions is given by $n v f_{o}$ is that post-collision molecules are selected from a Maxwellian distribution at the local average velocity and temperature. It is evident that this behavior can readily be incorporated in a particle code. By doing so, the burden of calculating collisions is reduced.

Despite the shortcomings of the BGK equation, it is able to reproduce the main features of the Boltzmann equation. Several modifications to the BGK equation have been proposed at the expense of the simplicity of the model. Cercignani (3) proposed a modification to the BGK equation that would allow it to reproduce the same viscosity and thermal conductivity as the full Boltzmann equation, here referred to as the BGKC method. To do this the Prandtl number is introduced as a parameter in the equation. The generalization of the BGK equation is obtained by the replacement of the Maxwellian distribution with a local anisotropic three-dimensional Gaussian, referred to as the ellipsoidal statistical (ES) model:

$$
f_{o}=\pi^{-3 / 2}(\operatorname{det} A)^{1 / 2} \exp \left[-\sum_{i, j=1}^{3} A_{i j} u_{i} u_{j}\right]
$$

where the matrix $A$ is:

$$
A_{i j}=\left[(2 R T / \operatorname{Pr}) \delta_{i j}-2(1-\operatorname{Pr}) p_{i j} /(\rho \operatorname{Pr})\right]^{-1}
$$

where $R$ is the universal gas constant, $\vec{u}$ is the velocity in the center-of-mass frame of reference, $\rho$ is the local density of the flow, $\operatorname{Pr}$ is the Prandtl number, and $p_{i j}$ is the pressure tensor. Recently this method was introduced into particle simulations with reasonable success (1).

\section{LANGEVIN'S EQUATION}

Langevin proposed a method for Brownian motion to model collisions between particles (4). According to this method, the influence of the surroundings on a particle that moves with a velocity $\vec{u}$ can be split up into two parts: a deterministic part representing the dynamical friction experienced by a particle $(-\beta \bar{u})$, and a probabilistic part characteristic of the Brownian motion $(\vec{U})$. The Langevin equation takes the form:

$$
\vec{u}=\vec{u}_{o} e^{-\beta \Delta t}+\vec{U}
$$

in which $\Delta t$ is a time interval over which motion occurs and $\vec{u}_{o}$ is the velocity of the particle at the beginning of the interval. The dynamical friction coefficient $\beta$ and the probabilistic part $\vec{U}$ are assumed to be independent of the velocity of the particle. The probabilistic part $\vec{U}$ is chosen from the modified Maxwellian distribution (4):

$$
P\left(\vec{u}, \Delta t, \vec{u}_{o}\right)=\left[\frac{m}{2 \pi k T\left(1-e^{-2 \beta \Delta t}\right)}\right]^{3 / 2} \exp \left[-\frac{m\left|\vec{u}-\vec{u}_{o} e^{-\beta \Delta t}\right|^{2}}{2 k T\left(1-e^{-2 \beta \Delta t}\right)}\right]
$$

in which $T$ is local temperature, $k$ the Boltzmann constant, and $m$ the mass of the particle. Effectively this distribution is Maxwellian at temperature $T\left(1-e^{-2 \beta \Delta t}\right)$. In this study the dynamic friction coefficient is interpreted as the equilibrium collision frequency.

It can be shown that the Langevin equation forms the basis for deriving all the physically significant relations concerning the motion of Brownian particles. The Langevin method has been applied successfully in the past for the modeling of charged flows with PIC by Jones et al. (5) and for DSMC by Gallis et al. (6). It should be noted, however, that the Brownian-motion paradigm corresponds more closely to the motion of charge particles in a plasma than to that of neutrals in rarefied flow. More specifically, Brownian motion describes the motion of a particle in very dense fluid (liquid density) in which multiple collisional interactions are very frequent, whereas the 
Boltzmann equation describes the motion of particles when only binary collisions are important. Thus, charged flows due to the long range interaction of the Coulomb forces fit the description of the Brownian motion more closely than neutral particles.

Another significant difference exists between the conventional DSMC collision operator and the Langevin model. In the DSMC method a fraction of the particles modify their properties significantly in every time step while the rest remain unchanged, but in the Langevin model every particle in a cell receives a small modification of its velocity in every time step. The modification constitutes a small change in their properties. It should be noted that the random velocities are not selected from a Maxwellian with the temperature $T$ of the background gas, but with a temperature of $T\left(1-e^{-2 \beta \Delta t}\right)$ that depends on the time interval and the collision frequency. In cases where the collision frequency is very low, the probabilistic contribution $\vec{U}$ adds a very small vector to the particle velocity. the deterministic contribution similarly applies a small modification to the particle velocity for every time step.

The Langevin method assumes that the thermal energy in a cell is always distributed according to a Maxwellian distribution. This assumption naturally restricts the method to equilibrium or near-equilibrium situations. The worst-case scenario for the method would be the collision of two monochromatic particle beams. While the DSMC method predicts a gradual depletion of particles from the initial distributions to an equilibrium distribution, the Langevin model evolves all particles gradually towards an equilibrium distribution at the average velocity and temperature.

\section{APPLICATION TEST CASE}

The first test case examined is that of the flow over a $10^{\circ}$ wedge (see Figures 1 and 2). The flow travels from left to right and the sides of the wedge are assumed to be specularly reflecting. The gas is pure argon at an ambient temperature of $180 \mathrm{~K}$ and a number density of $10^{22} \mathrm{~m}^{-3}$ with an upstream velocity of $1365 \mathrm{~m} / \mathrm{s}$ (see Table 1). The domain is discretized with 25,000 cells, and a time step of $10^{-8} \mathrm{~s}$ is used. The downstream boundary condition is assumed to be non-reentrant (vacuum), which is acceptable for the supersonic flow downstream of an oblique shock. This test case is simulated with the DSMC, BGKC and Langevin methods. Steady-state conditions are observed after 5,000 time steps while the code executes a total of 65,000 time steps.

\begin{tabular}{|l|l|}
\hline \multicolumn{2}{|c|}{ Upstream Conditions } \\
\hline Molecular Mass & $39.99 \mathrm{amu}$ \\
\hline Specific Heat Ratio & $5 / 3$ \\
\hline Number Density & $10^{22} \mathrm{~m}^{-3}$ \\
\hline Temperature & $180 \mathrm{~K}$ \\
\hline Speed of Sound & $249.75 \mathrm{~m} / \mathrm{s}$ \\
\hline Free Stream Velocity & $1365 \mathrm{~m} / \mathrm{s}$ \\
\hline Mach Number & 5.465 \\
\hline \multicolumn{2}{|c|}{ Downstream Conditions } \\
\hline Number Density & $2.1 \times 10^{22} \mathrm{~m}^{-3}$ \\
\hline Temperature & $336 \mathrm{~K}$ \\
\hline Speed of Sound & $341 \mathrm{~m} / \mathrm{s}$ \\
\hline Velocity & $1304 \mathrm{~m} / \mathrm{s}$ \\
\hline Mach Number & 3.823 \\
\hline Wedge angle & $10^{\circ}$ \\
\hline Shock angle & $19.55^{\circ}$ \\
\hline
\end{tabular}

Table 1. Shock wave conditions

Figure $1(\mathrm{a}-\mathrm{c})$ presents the number-density contour lines as calculated by the DSMC, BGKC and Langevin methods. Figure $2(\mathrm{a}-\mathrm{c})$ in the same fashion presents the corresponding translational temperature contour lines. At $x$ $=y=0$, the location of the leading edge of the wedge, the shock layer forms.

A quantitative comparison indicates that all three simulations are in agreement about the downstream conditions and the shock angle. For example, with all three methods, the flow downstream eventually reaches a maximum number density that is about double the upstream number density, and the shock angle is $19-20^{\circ}$, as 
theoretically predicted (see Anderson (7)). The fact that the DSMC, BGKC and Langevin solutions produce the same downstream temperature and number density confirms that all methods conserve momentum and energy.

Comparing the figures in more detail, it is seen that the Langevin method predicts a very thin shock compared to the DSMC and BGKC solutions, which are seen to be in remarkable agreement throughout the domain. The temperature increases from $180 \mathrm{~K}$ upstream to $336 \mathrm{~K}$ in the downstream area. For the DSMC and BGKC methods, only a small portion of the downstream flow reaches the equilibrium temperature, while the Langevin solution produces a large downstream area at a temperature of $336 \mathrm{~K}$.

The shock thickness depends on the collision frequency: a larger collision frequency produces a thinner shock. The reason for this excessive collisionality of the Langevin model is that it describes the Brownian motion, in which multiple collisions are very frequent. This differs significantly from the paradigm of a dilute gas in which only binary collisions are important.

An attempt was made to circumvent this problem by artificially decreasing the collision frequency. By multiplying the collision frequency by a factor of 0.25 , the correct shock thickness can be reproduced by the Langevin method for this particular problem. Unfortunately, this factor was found to be very problem-dependent. Therefore, such a modification does not have any physical validity and cannot be recommended.

\section{CONCLUSIONS}

From this comparison, it is clear that the Langevin approach overestimates the collisionality of the flow with respect to the DSMC and BGKC methods. As mentioned earlier, the paradigm of the Langevin approach is quite different from that of the Boltzmann equation. The Langevin approach may be suitable for very high densities and charged flows, in which the collisions are so frequent that they can be replaced by a Brownian force acting on the particles. However, its application to dilute gases does not appear to be appropriate.

\section{ACKNOWLEDGEMENTS}

The authors would like to thank Dr. Wahid L. Hermina, Dr. Matthew M. Hopkins and Dr. Richard J. Buss of Sandia National Laboratories for their helpful comments. This work was performed at Sandia National Laboratories. Sandia is a multiprogram laboratory operated by the Sandia Corporation a Lockheed Marin Company, for the United States Department of Energy under Contract DE-AC04-94AL85000.

\section{REFERENCES}

1. Gallis M.A. and Torczynski J.R. "The Application of the BGK Model to Particle Simulations," AIAA paper 2000-2360, 34th Thermophysics Conference, Denver Co. 2000.

2. Vincenti W.G. and Kruger C.H., Introduction to Physical Gas Dynamics, Krieger Publishing Co, 1986.

3. Cercignani C., The Boltzmann Equation and its Applications, Springer-Verlag, 1988.

4. Reif, F., Fundamentals of Statistical and Thermal Physics, McGraw Hill, 1965.

5. Jones M.E., Lemons D.S., Mason R.J., Thomas V.A. and Wiske D., "A Grid Based Coulomb Collision Model for PIC Codes," Joumal of Computational Physics 123, 169-181, 1996.

6. Gallis M.A. and Harvey J.K., "Modelling of Ionised Flows in DSMC," Proceedings of RGD 21, 1998.

7. Anderson J.D., Modern Compressible Flow, McGraw-Hill, 1990. 


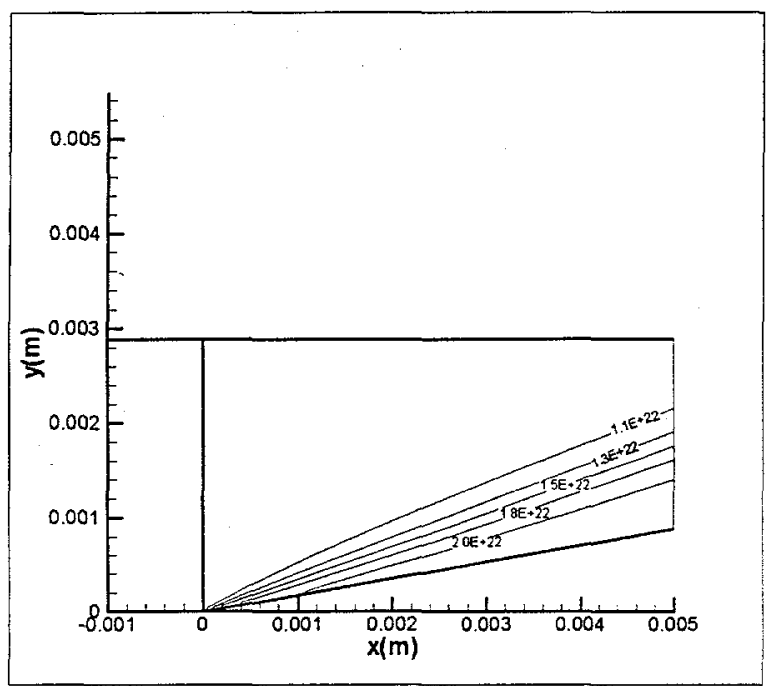

Figure 1a. DSMC: number density

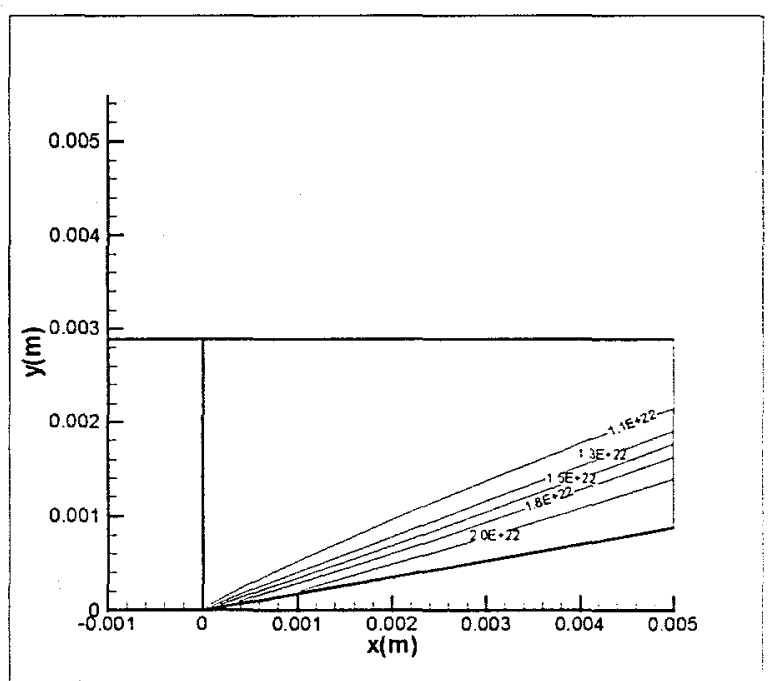

Figure 1b. BGKC: number density

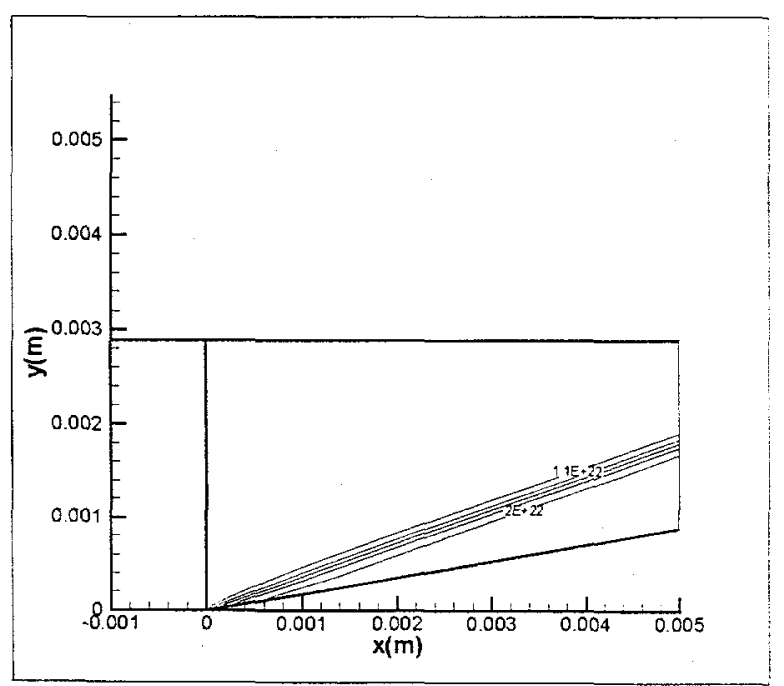

Figure 1c. Langevin: number density

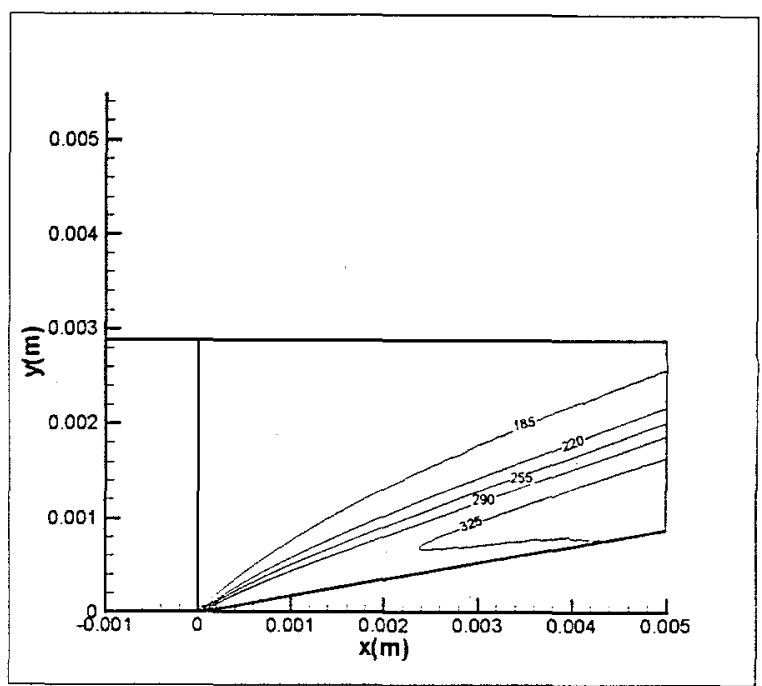

Figure2a. DSMC: translational temperature

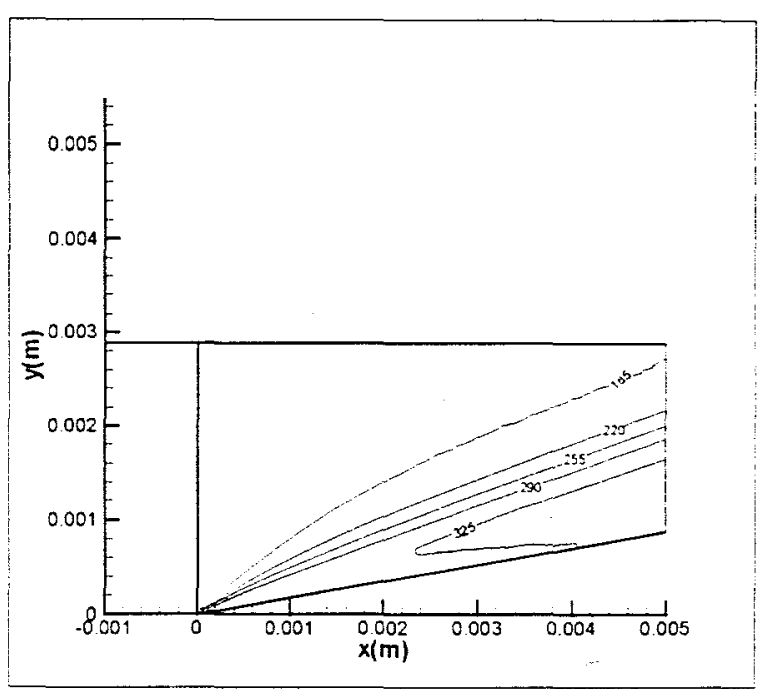

Figure 2b. BGKC: translational temperature

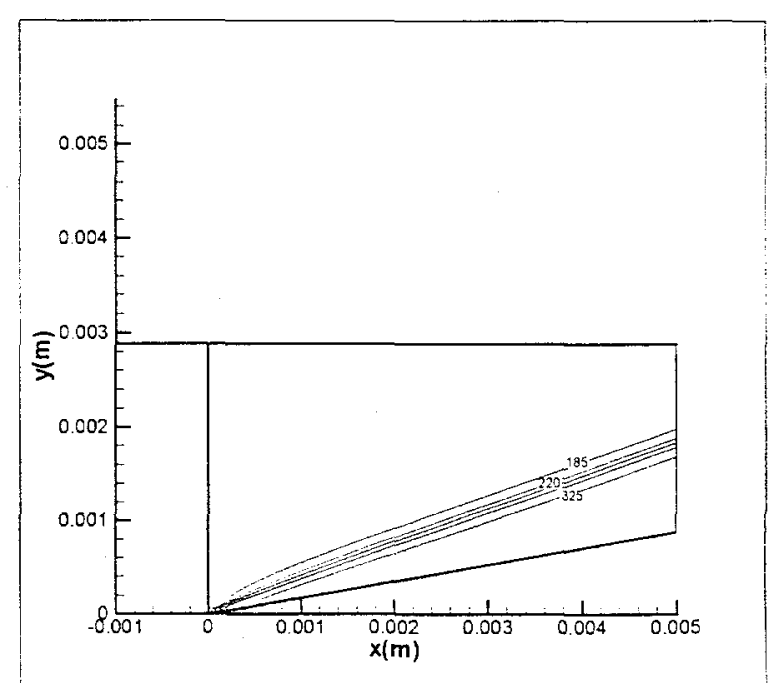

Figure 2c. Langevin: translational temperature 